\title{
TWO-PHASE MOBILE INTERCONNECTION SCHEMES FOR ULTRA-GRAIN PIPELINE APPLICATIONS
}

\author{
Juan Núñez , María J. Avedillo and José M. Quintana \\ Instituto de Microelectrónica de Sevilla (IMSE-CNM-CSIC) \& Universidad de Sevilla
}

Av. Américo Vespucio s/n, 41092, Sevilla (Spain)

\{jnunez, avedillo,josem\} @ imse-cnm.csic.es

\begin{abstract}
Monostable to Bistable (MOBILE) gates are very suitable for the implementation of gate-level pipelines which can be achieved without resorting to memory elements. MOBILE operating principle is implemented using two series connected Negative Differential Resistance (NDR) devices with a clocked bias. This paper describes and experimentally validates a two-phase clock scheme for such MOBILE based ultra-grain pipelines. Up to our knowledge it is the first MOBILE working circuit reported with this interconnection architecture. The proposed interconnection architecture is applied to the design of a 4-bit Carry Lookahead Adder.
\end{abstract}

Keywords: Negative Differential Resistance (NDR), Nanopipeline, Monostable to Bistable Logic Elements (MOBILE).

\section{INTRODUCTION}

Different emerging devices like Resonant Tunneling Diodes (RTDs), tunnel transistors or molecular RTD devices exhibit Negative Differential Resistance (NDR) in their $I-V$ characteristic. Many circuits taking advantage of it have been reported covering different applications and with different goals including high speed, low power or reduced device 
count [1], [2], [3] so that design techniques exploiting this feature at difdifferent levels (circuit, architecture, ..) are currently an area of active research.

From the design point of view, the NDR characteristics are very attractive. On one hand, it can be exploited in non-linear circuits like oscillators or frequency dividers. On the other, it is useful in the implementation of memories due to the existence of stable states associated to the inclusion of NDR elements. In particular, the Goto pair [4] is well known. The circuit consists of two NDR devices connected in series leading to three operating points, two stable and one unstable. The two stable points can be used to represent and store data.

On the basis of the Goto pair, logic circuits which operation is based on a Monostable to a Bistable transition (MOBILE) have been developed. MOBILE gates are implemented operating two series connected NDR devices with a switching bias. There are two interesting characteristics of MOBILE gates in comparison to conventional logic gates implementations.

First, they increase the functionality implemented by a single gate in comparison to MOS and bipolar technologies thus reducing circuit complexity. In particular, the operating principle of MOBILE is extremely well suited to implement the arithmetic operation on which Threshold Gates (TGs) are based [5]. Different topologies for RTD TGs and MultiThreshold Threshold gates have been reported and experimentally validated.

Second, the latching property of MOBILEs arising from their NDR characteristic allows the implementation of gate-level pipelines which can be achieved without resorting to memory elements [1], [6] , and which do not exhibit the functional limitation of conventional CMOS solutions based on dynamic logic, allowing only non-inverting blocks to be chained.

Originally, it was proposed to operate MOBILE gates in a gate level pipelined fashion using a four-phase clock scheme. However, operating frequency (or throughput) depends not only on the number of gate levels, but also on the number of clock-phases, since clock period needs to accommodate all the phases. Thus, from the point of view of speed a twophase scheme is very attractive.

This paper explores in depth and experimentally validates a two- 
phase clock scheme for MOBILE based fine-grain pipelines.

The paper is organized as follows: in Section II, MOBILE logic style is described. In Section III, two-phase gate-level MOBILE pipelines are introduced, showing experimental results that validate their operation. In Section IV a Carry Lookahead Adder which has been designed using the proposed pipeline is described. Finally, some key conclusions are given in Section V.

\section{BACKGROUND}

\subsection{MOBILE LOGIC GATES}

The MOBILE [5] in Fig. 1a is an edge-triggered current controlled gate which consists of two devices exhibiting NDR in their $I-V$ characteristic (Fig. 1b), connected in series and driven by a switching bias voltage, $V_{C K}$. When $V_{C K}$ is low, both NDRs are in the on-state and the circuit is monostable. Increasing $V_{C K}$ to an appropriate maximum value ensures that only the device with the lowest peak current switches from the onstate to the off-state. Output is high if the driver NDR switches and it is low if the load does. Logic functionality can be achieved if the peak current of one of the NDR devices is controlled by an input. In the configuration of the rising edge-triggered inverter MOBILE shown in Fig. $1 \mathrm{c}$, the peak current of the driver NDR can be modulated using the external input signal $V_{i n}$. Transistor behaves like a switch, so that for a low input, current flows only through $N D R_{D}$, but for a high input, the effective peak current of the driver is the sum of the peak currents of $N D R_{D}$ and $N D R_{X}$. Replacing the single transistor in Fig. 1c by an NMOS transistor network, other logic functions are implemented. NDR peak currents are selected such that the value of the output depends on whether the network transistor evaluates to "1" or to "0". Figure $1 \mathrm{~d}$ depicts a falling edge triggered inverter. Note that branch implementing functionality is now in parallel to the load NDR and uses a p-type transistor. 


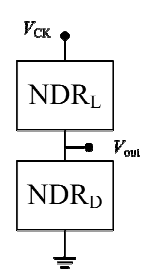

(a)

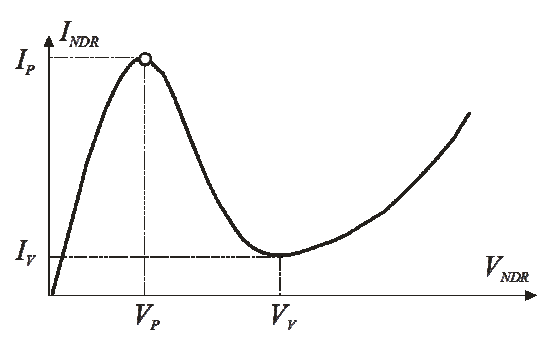

(b)

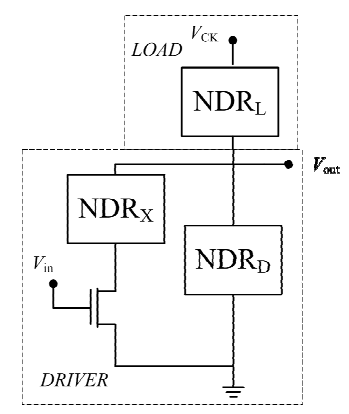

$(c)$

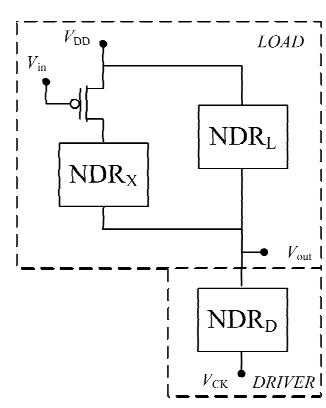

$(d)$

Fig 1. MOBILE circuits. (a) NDR I-V characteristic. (b) Basic MOBILE. (c) Rising edge-triggered MOBILE inverter. (d) Falling edge-triggered MOBILE inverter.

A sufficiently slow $V_{C K}$ rising (or falling) is required for MOBILE operation [7]. That is, there is a critical rise time for the switching bias below which the gate does not operate correctly. Under that critical rise time, there is at least one input combination for which the gate does not produce the expected logic output. Since AC currents associated to internal parasitics and output capacitive loads (fan-out) are more important for faster clock changes, the ideal MOBILE operating principle, based on peak currents comparison, can be substantially modified. This critical value depends on both circuit (NDR peak currents, fan-out ...) and technological parameters. That is, design requires taking into account these AC currents in order to guarantee the desired relationship between load and 
driver currents for each input combination when $V_{C K}$ approaches $2 V_{p}$, bebeing $V_{p}$ the peak voltage of the NDRs (see Fig. 1a).

Rising (falling) edge-triggered MOBILE logic gates evaluate the inputs with the rising (falling) edge of the bias voltage and hold the logic level of the output while the bias voltage is high (low), even though the inputs change (self-latching operation [8]). The output returns to zero (to one) with the falling (rising) edge of the clock until the next evaluation. The self-latching operation allows the implementation of gate-level pipelined architectures without extra memory elements [1] and without the functional limitations of dynamic based solutions like the widely used domino logic style.

\subsection{INTERCONNECTING MOBILE GATES}

As it was stated in previous section, and assuming rising-edge MOBILEs, there are four steps in the operation of each gate: evaluation (clock rises),
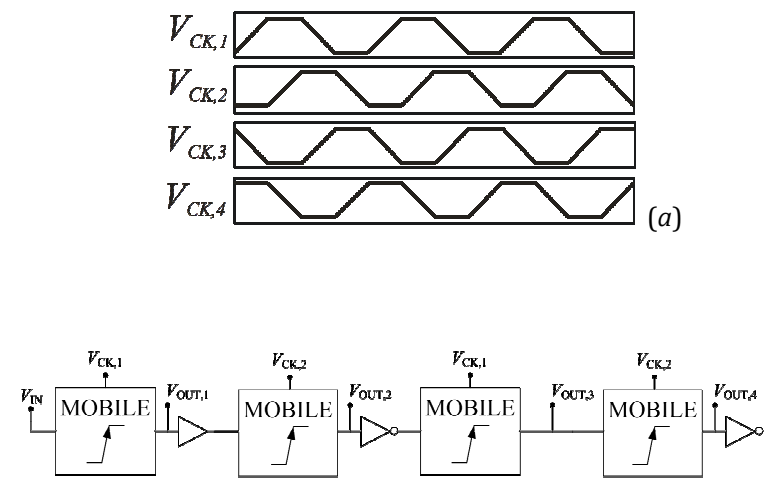

(b)

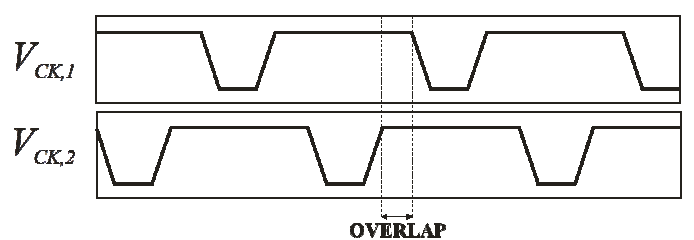

$(c)$

Fig 2. (a) Four-phase clock scheme. (b)-(c) Block diagram and clock waveforms of the two-phase chain of inverters. 
hold (clock high), reset (clock falls) and wait (clock low). Gate-level pipepipelining is possible if each MOBILE gate evaluates while those driving it are in the hold state. In this way, it is guaranteed that inputs to each gate are stable during evaluation, and that the reset of the MOBILE gates does not affect those they drive, since they have already evaluated when it happens. Thus, memory elements are not required. Note that this is true both for inverting and non inverting MOBILE stages, and even when adding an output stage (static inverter or buffer) to the output of the MOBILE blocks to ease management of fan-out and interconnections. That is, fulfilling above stated constraint allows ultra fine-grain pipeline operation where both inverting and non inverting stages are allowed. In domino solutions only non inverting stages are possible which complicates logic design (inverters need to be pushed towards the inputs or some parts of the circuits are duplicated), unless a double rail implementation is used which almost duplicates device count.

Conventionally, and because of the four steps in MOBILE operation, cascaded rising edge-triggered MOBILE gates are operated in a pipelined fashion using a four-phase overlapping clocking scheme shown in Fig. 2a [1]. $V_{C K, i}$ is delayed with respect to $V_{C K, i-1}$ by $T / 4$, being $T$ the clock period. In this way the $i^{\text {th }}$ stage evaluates (rising edge of $\left.V_{C K, i}\right)$ while the $(i-1)^{\text {th }}$ stage is in the hold phase $\left(V_{C K, i-1}\right.$ high). Four clock signals are enough, since the first phase can be used for the fifth level and so on. In previous section, it was stated that there is a critical rise time below which the gate does not operate correctly. This explains the clock shape with equal rise, high, fall and low times. Thus, for this scheme four gates/stages serially evaluate in one clock period.

However other schemes are compatible with the constraint that one stage evaluates while preceding stages are in hold state. Moreover, the constraint can be somewhat relaxed making possible other simpler schemes.

Single phase scheme has been proposed [9]. However there are two drawbacks associated to the single-phase solutions. First, negative edgetriggered MOBILE are used which requires p-type transistors. This translates in larger transistors and so in larger parasitic capacitances 
which degrade gate speed. Second, they exhibit limited clock-skew tolertolerance. Recently, we have proposed a two-phase scheme [10] which overcomes both issues while being similar in terms of throughput and latency.

Next section describes the two-phase interconnection scheme and shows experimental results of fabricated circuits validating the approach.

\section{TWO-PHASE MOBILE NETWORKS}

An alternative solution consists of the design of networks of only positive edge triggered MOBILE gates operated with an overlapping two phase clock scheme as shown in Figures. $2 \mathrm{~b}$ and $2 \mathrm{c}$. It can be easily seen that each gate evaluates while preceding one is in the hold state, and that only two stages serially evaluates in one clock period. Note that inter-gate elements (inverting or not inverting) can also be added if required by logical (to increase design flexibility), or electrical (for example, efficient handling of large loads) considerations.

It is interesting to make some comments concerning the amount by which the clock-phases overlap. Due to the edge-triggered nature of MOBILE evaluations, required minimum overlap is generally small, especially when inverters/buffers are used between MOBILE blocks, since, as it was anticipated, the interconnection constraint can be relaxed. What is required for proper operation is that current stage takes a decision before it sees the reset of the previous stage. That is, before the output of the preceding MOBILE blocks reaches a low level output voltage and it propagates through the inter-MOBILEs elements. The maximum overlap is only limited by the maximum allowable duty cycle of the clock, which is determined by the minimum time required for the reset of the MOBILE gates. MOBILE output must discharge to zero before an evaluation. Thus, overlap is fixed such that clock skew is tolerated.

Two-phase clocked chains of MOBILE inverters have been designed and fabricated. These structures have been implemented with MOS-NDR devices (circuits made up of transistors that emulate the NDR I-V characteristic) and the MOBILE gate topology from [11] in a $1.2 \mathrm{~V} / 90 \mathrm{~nm}$ CMOS technology. Figures $4 \mathrm{a}$ and $4 \mathrm{~b}$ depict the schematics of the MOS- 
NDR device which has been used and the schematic of a MOBILE inverter implemented with them. The design of MOBILE blocks operating at high frequencies is not straightforward. As it was previously mentioned, it is necessary to take into account AC currents associated to parasitics which can be large at high frequencies. Thus, design validation requires both an accurate modeling of layout parasitics and experimental validation.

Figure 4c depicts the block diagram of one of the fabricated circuits. Each MOBILE stage is an inverter similar to the one depicted in Fig. 4b. As shown in Fig. 4c, a two-phase clock generator has been also included. It provides two non-overlapped clock signals $\left(V_{C K, 1}\right.$ and $\left.V_{C K, 2}\right)$ with the same frequency of the master clock $\left(V_{C K}\right)$. Note that power clocks are avoided since the clock signal of each MOBILE circuit is applied to the input of a static inverter. The output of this inverter is used as the clock of the MOBILE inverter. In this way, the two required overlapped clocks are generated and the constraints on clock rising time are relaxed.

The packaged circuit has been probed and correct operation has been shown. Figure $4 \mathrm{~d}$ depicts experimental results when a sequence alternating 0's and 1's is applied to the ten-stage pipeline. Waveforms of the master clock, the input $\left(V_{I N}\right)$ and the output $\left(V_{\text {OUT }}\right)$, which have been captured using the oscilloscope Agilent DS06104A, are shown. Note that, in addition to package, there are input buffers (for $V_{I N}$ and $V_{C K}$ ), output buffers and pads which are not shown in Fig. $4 . V_{C K}$ and $V_{I N}$ are $1.2 \mathrm{~V}$ pulse trains at $1 \mathrm{GHz}$ and $500 \mathrm{MHz}$, respectively. As expected, $V_{\text {OUT }}$ is a periodical signal of the same frequency of the input. The $0101 . . .01$ sequence is obtained at the output of the pipeline with a latency of five clock cycles, since data is evaluated twice each cycle of $V_{C K}$. Note the different shapes of $V_{I N}$ and $V_{\text {OUT }}$ which is due to the return to zero behavior of MOBILE. Results are shown at $1 \mathrm{GHz}$ so that signals are attenuated by the experimental set-up. Finally, Fig. 4e shows the output of another twophase chain of inverters incorporating static buffers between MOBILE stages. For this circuit, both $V_{C K}$ and $V_{I N}$ have been generated on- chip so that the input frequency is half that of the clock $(1.7 \mathrm{GHz})$. 

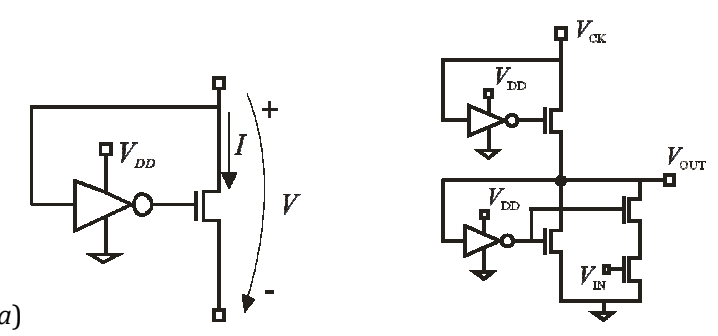

(b)

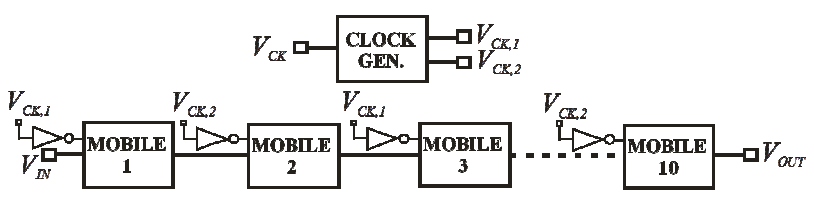

$(c)$

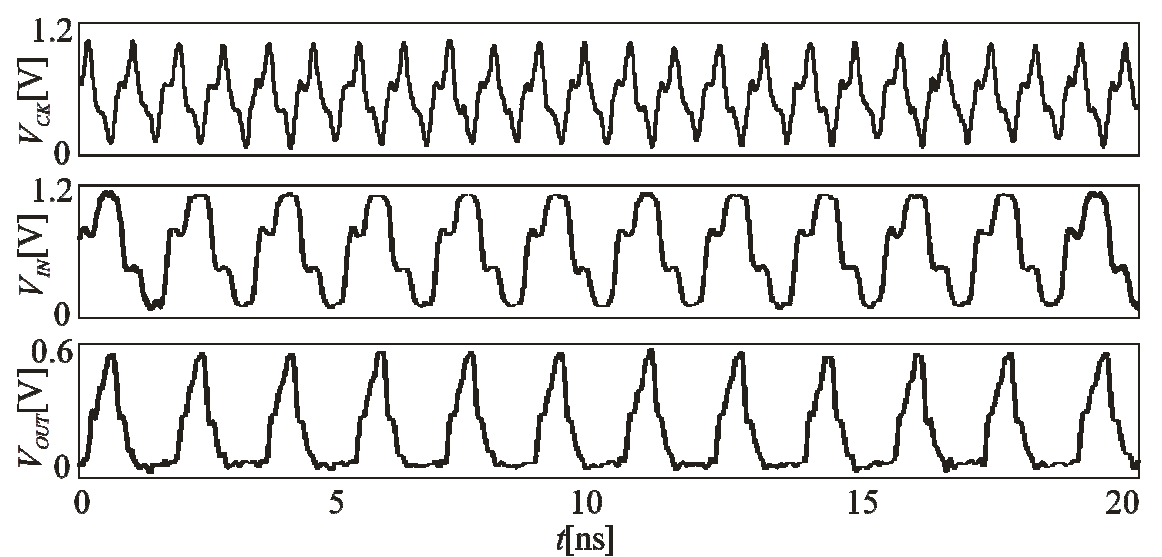

$(d)$

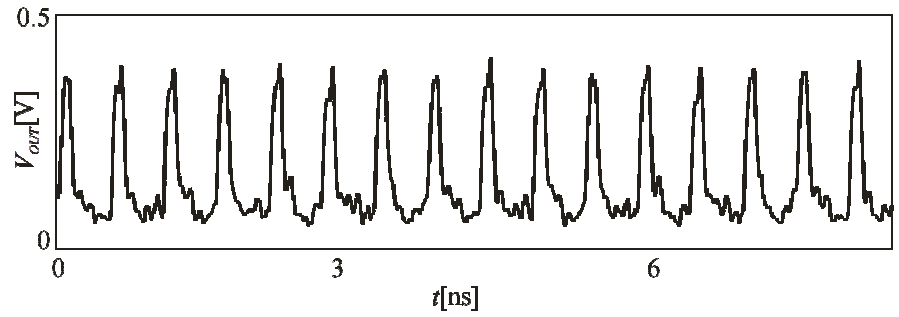

$(e)$

Fig 4. (a) MOS-NDR device. (b) MOBILE inverter implemented with MOS-NDR devices. (c) Block diagram of a fabricated two-phase chain of inverters. $(d)$ Measured waveforms. $(e)$ Output of a fabricated two-phase chain of inverter with static buffers as interconnection stages. 


\section{4-BIT CLA}

The two-phase clock scheme has been applied in the design of a 4-bit RTD-CMOS MOBILE Carry Lookahead Adder (CLA) as a case study. Figure 5 a shows the block diagram of a 4-bit CLA. The PGs blocks propagate $\left(P_{i}=\right.$
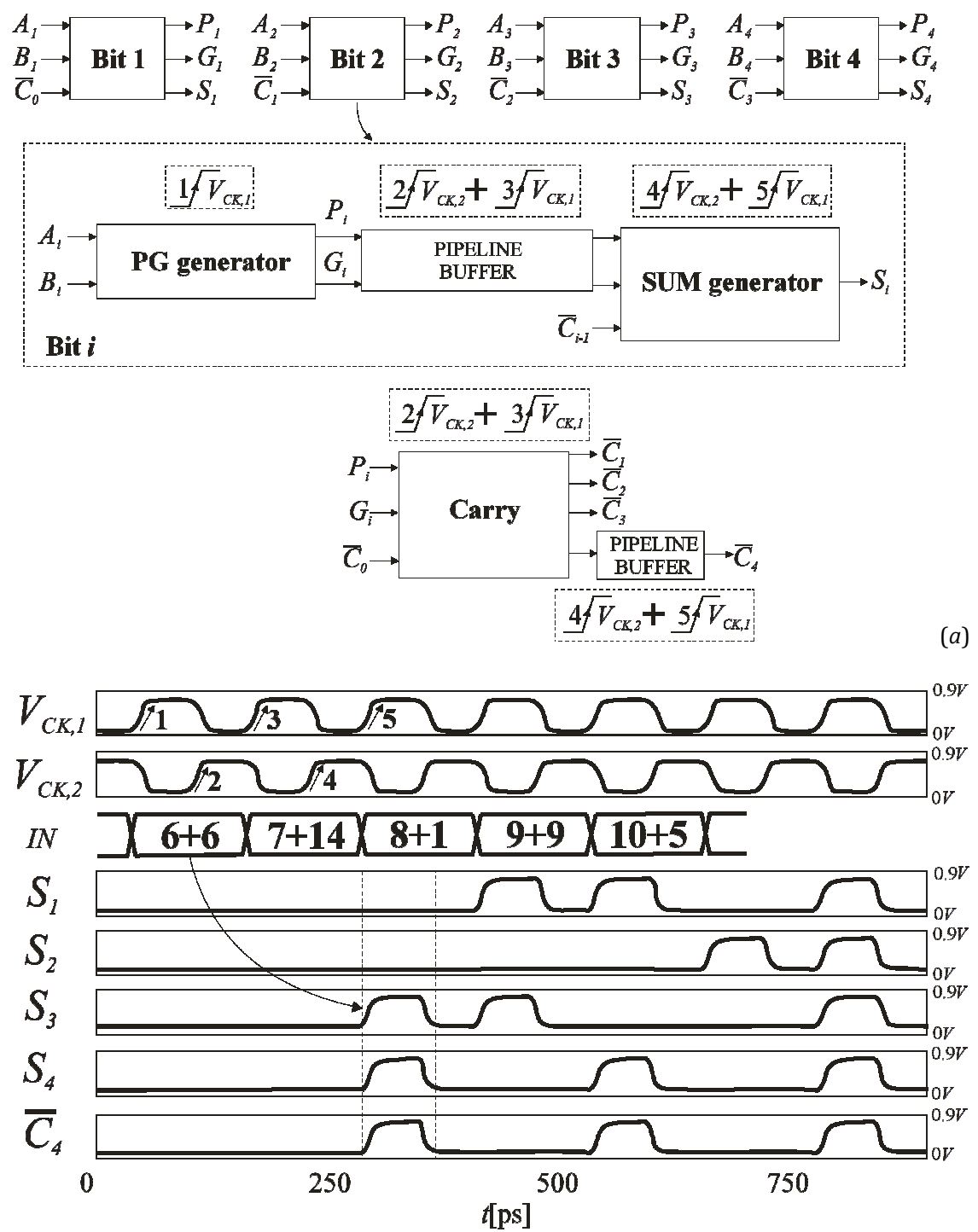

(b) 
$A_{i}$ XOR $\left.B_{i}\right)$, generate $\left(g_{i}=A_{i} A N D B_{i}\right)$ and sum $\left(S_{i}=A_{i}\right.$ XOR $B_{i}$ XOR $\left.C_{i}\right)$ bits. The Carry block generates the carry signals $C_{1}, C_{2}, C_{3}$ and $C_{\text {out }}\left(C_{i+1}=G_{i}\right.$ OR $\left(P_{i}\right.$ AND $C_{i}$ ). The carry block is implemented with two pipeline stages using NOR gates. Implementation of XOR gates takes advantage of the possibility of using inverting and non inverting inter-stages elements so that variables and their complements are available. There are five stages in the design and so latency is two-cycles and a half. In Fig. 5a it has been marked which clock cycles corresponds to each stage.

The study uses PTM 32nm transistor model. The RTD has been modeled using a voltage-dependent current source and a capacitor in parallel and technology parameters from an experimentally validated Si-Ge RTD [8] with peak current density $j_{p}=218 \mathrm{KA} / \mathrm{cm}^{2}$ and capacitor $C=6 \mathrm{fF} / \mu \mathrm{m}^{2}$. Transistor lengths have been set to the minimum value associated to the technology whereas their widths are large enough to allow their operation as switches. RTD areas have selected to work at a frequency of $0.12 / F O-4$ (FO-4 is the FanOut-4 inverter delay of the technology, $14.69 \mathrm{ps}$ ) at a supply voltage of $0.9 \mathrm{~V}$. Parasitics capacitances have been added to model drain and source diffusion parasitic (0.25fF), RTDtransistor contacts $(0.25 \mathrm{fF})$ and interconnections $(1 \mathrm{fF})$.

Figure $5 b$ shows simulation waveforms of the designed CLA for selected values of the inputs $(A+B)$, where correct operation is observed. Note that both the sum bits and the carry are obtained after the third rising edge of $V_{C K, 1}$ (two-cycles and a half from the evaluation of the inputs).

\section{CONCLUSIONS}

The operation of two-phase gate-level pipelines based on MOBILE operating principle has been experimentally validated. Up to our knowledge it is the first time a working two-phase MOBILE network is reported. This interconnection scheme has advantages over other previously reported clock schemes for MOBILE logic networks. It is simpler than the conventional four-phase solution and leads to higher operating frequencies since only two-stage, instead of four, sequentially evaluates in one clock cycle. Unlike one phase scheme, p-type transistors are avoided and clock skew tolerance increases. The later is due to the 
self-latching property of MOBILE gates which makes possible an operaoperation taking advantage of the overlapping of the two clock phases to tolerate clock skew and, in addition, avoids limitations of conventional CMOS counterparts. The design of a two-phase 4-bit RTD-CMOS CLA has been carried out as an application example.

Acknowledgement. This work has been funded by Ministerio de Economía y Competitividad del Gobierno de España with support from ERDF under Project TEC2010-18937.

\section{REFERENCES}

[1] P. Mazumder, S. Kulkarni, M. Bhattacharya, J.P. Sun, and G.I. Haddad, "Digital circuit applications of resonant tunneling devices", Proc. IEEE, 86, pp. 664-686, Apr. 1998.

[2] S. Choi , Y. Jeong, J. Lee, and K. Yang, "A Novel High-Speed Multiplexing IC Based on Resonant Tunneling Diodes”, IEEE Trans. On Nanotechnology, vol. 8, no. 4, pp. 482-486, Jul. 2009.

[3] K. Karda, J. Brockman, S. Sutar, A. Seabaugh, and J. Nahas, "Bistable-Body Tunnel SRAM”, IEEE Trans. Nanotechnology, vol. PP, no. 9, pp. 1, 2010.

[4] E. Goto, K. Murata, K. Nakazawa, K. Nakagawa, T. Moto-Oka, Y.Ishibashi, T. Soma, and E. Wada, "Esaki diode high-speed logical circuits," IRE Trans. Elect. Comp., vol. EC-9, pp. 25-29, 1960.

[5] T. AKeyoshi, T., et al., "Weighted sum threshold logic operation of MOBILE's (Monostable Bistable Logic Element) using resonant-tunnelling transistors", IEEE Electron Device Lett., 14, pp. 475-477, Oct. 1993.

[6] S. Mohan, et al., "Ultrafast pipelined arithmetic using quantum electronic devices", Computers and Digital Techniques, IEE Proceedings, 141, (2), pp.104-110, Mar 1994.

[7] H. Matsuzaki, H. Fukuyama and T. Enoki: "Analysis of Transient Response and Operating Speed of MOBILE”, IEEE Trans. on Electron Devices, Vol. 51, no. 4, pp. 616-622, April 2004.

[8] M.J. Avedillo, J.M. Quintana and H. Pettenghi, "Self-Latching Operation of MOBILE Circuits using Series-Connection of RTDs and Transistors", IEEE Trans. on Circuits and Systems-II, Vol. 53, no. 5, pp. 334-338, May 2006.

[9] J. Núñez, M.J. Avedillo and J.M. Quintana, "Simplified single-phase clock scheme for MOBILE networks”, Electronics Letters, Vol. 47, no. 11, pp. 648-650, May 2011.

[10]J. Núñez, M.J. Avedillo and J.M. Quintana, “Domino Inspired MOBILE Networks”, Electronics Letters, Vol. 48, no. 5, Feb, 2012.

[11]J. Núñez, M.J. Avedillo and J.M. Quintana, "Efficient Realisation of MOS-NDR Threshold Logic Gates", IET Electronics Letters, Vol. 45, no 23, pp. 1158-1160, 2009. 
[12] S.Y. Chung, et al, "Si/SiGe resonant interband tunnel diode with fro $20.2 \mathrm{GHz}$ and peak current density $218 \mathrm{kA} / \mathrm{cm} 2$ for K-band mixed-signal applications". IEEE Electro Device Lett., vol 27, pp. 364-367, 2006. 